\title{
The impact of Tesla's bitcoin investment and its plans to accept it as payment method on the evolution of bitcoin
}

\author{
Alexandra MIRONEANU \\ International Master of Business Administration, Bucharest, Romania \\ alexandra.miroaneanu@gmail.com \\ Beatrice IRIMIA \\ International Master of Business Administration, Bucharest, Romania \\ beatriceirimia123@yahoo.com \\ Valentina SĂNDULESCU \\ International Master of Business Administration, Bucharest, Romania \\ valentina.marlene@gmail.com \\ Casiana TEODOROIU \\ International Master of Business Administration, Bucharest, Romania \\ teodoroiu.casiana@yahoo.com
}

\begin{abstract}
For the past years, cryptocurrencies have been a hot and controversial topic that has captured the attention of the whole tech world. Even if right now the portfolio of digital assets is considerable in size, the first cryptocurrency, Bitcoin, exploited its pioneer advantage and managed to remain at the center of attention both for the media and investors.

During 2020, one of the most chaotic years, Bitcoin boomed in November 2020 almost doubling since the end of 2019. This boom is the result of a combination of factors, such as the fear of missing out translated into a chain reaction of public and private companies to consider Bitcoin a safe reserve asset, a hedging method against inflation, which represents a substitute for traditional hedging instruments, the infrastructure developed around it over the years, and lastly the hype created by influential figures through news and social media platforms. There have been many public figures that exhibited interest in cryptocurrencies through platforms such as Twitter, for instance Elon Musk, Bill Gates, Kanye West, Hugh Laurie, Mike Tyson and Gwyneth Paltrow are just some in a long list of celebrities that backed Bitcoin.

This paper aims to analyze the impact that twitter posts have upon the evolution of Bitcoin, coupled with Tesla's investment and recent statement of introducing Bitcoin as a method of payment in the near future. Our research tries to determine if the news and social media posts, such as tweets have an influence upon Bitcoin's volatility and fluctuations.
\end{abstract}

Keywords: Cryptocurrency, Bitcoin, Tesla, social media, Twitter, volatility.

\section{Introduction}

2009 is the year that marks the creation of a decentralized "peer to peer" financial system called Bitcoin, by Satoshi Nakamoto, a Japanese programmer. Although not original, since the "cryptocurrency" concept first appeared in 1998, being introduced by Wei Dai (Okhuese, 2017), Bitcoin is considered to override the fiat currency in various aspects, given its transnational dimension, as it can be transferred across borders bereft of restrictions. Further, its growing appeal to investors all over the world is backed by the fact that transactions bear modest fees while its transparency is derived from the access of users to the public ledger. As previously mentioned, Bitcoin, is a 
decentralized virtual currency blueprint characterized by a bidirectional flow, together with a cryptocurrency. It was conceived so as to be bank, government, or any other institution independent. Largely, Bitcoin was a similar working mechanism with electronic cash. The cryptocurrency can be acquired from different platforms/websites, exchanged for the domestic currency, with an exchange rate driven by the market, as a result of the supply and demand function. The software used for payments is known as "wallet", and in the occurrence of a payment the account of the sender is debited while the account of the recipient is credited respectively. (Segendorf, 2014). By and large, Bitcoin is covered by the veil of volatility, coupled with the degree of people's acceptance, which is bound to diminish, given the increasing number of users adhering to the network (Kaushal et al., 2017).

At the outset, Bitcoin had a rather sluggish start and only captured traction when media started providing reports on its valuation, which led to a frenzy that helped it gain more and more attention. The buzz created around its upscaled, tech-savvy appeal, thus contributed to the "unrealistic" valuation of its price and its connected volatility (Leath, 2019). Notwithstanding, given the extreme nature of its volatility, Bitcoin is considered a speculative investment, and not necessarily a currency (Chena at el., 2020). Bitcoin's volatility represents the extent to which the price changes from a period to another, which leads to risk when it comes to holding it, since its value may rise dramatically or plummet substantially. During 2009 up to 2011, Bitcoin's price was appreciated to be stable, yet following 2011, the price began to fluctuate reaching a value of US $\$ 32$ from US $\$ 0.30$ dollars, then it flattened to 2 dollars, and it reached $\$ 13$ by December 2012. In April next year, Bitcoin was traded at $\$ 220$, yet it plummeted to approximately $\$ 70$ in the same month. In the following years, the price would surge and collapse in a matter of months or even weeks. In November 2017, the price reached the milestone of $\$ 10$ 000, while one month later, in December, one Bitcoin reached roughly $\$ 20000$ (Davis and Petasis, 2021). In 2018, one Bitcoin coin was valued at approximately $\$ 19000$ and this extensive rise matched enormous public interest, specifically the investors profits which arose from the cryptocurrency' s rise. During the last months of the year, Bitcoin faced a dramatic plummet as it decreased with about $80 \%$ of its value, one Bitcoin reaching a value of $\$ 3$ 800. Numerous individuals lost the majority of their Bitcoin investment by the end of the year and claimed to have stopped using the crypto currency given the exposure to the monetary risk.

Several Bitcoin disincentives list the trust in the prospects of the next few years. Trust is the key to functioning for Bitcoin, as it requires trust regarding the transactional partners, in its capability as a "shared value store" and in its technology (Work, 2019). Despite prevailing issues, it nonetheless has a database of active users who perform thousands of daily transactions together with numerous online communities that appreciate the merits of the currency such that, in February 2021, Bitcoin price is approximately $\$ 55000$ (YahooFinance, 2021).

During the last period Bitcoin attracted a number of investment funds and publicly traded companies, marking a shift in perception and making an increasing number of people buy the crypto currency. Examples include companies such as: Square, Galaxy Digital Holdings, Hut 8 MiNING, Voyager Digital (Forbes, 2021). MicroStrategy, a platform keen on business analytics, also embraced Bitcoin and owns an equivalent of around $\$ 3.6$ billion and is said to borrow $\$ 600$ million to acquire even more of the currency (Reuters, 2021) .

Much as the research in behavioral economics advocates that emotions have the possibility to alter individual behavior together with process of decision-making, Twitter, the platform for microblogging, has been increasingly attracting the attention of multiple fields of study, being perceived to analyze economic and social data sets. Interestingly, the platform seems to have an 
influence on its users and on the diffusion of information. (Kaminski, 2016). Research on this topic has shown there is a correlation between the activity related to "internet message boards" and the volatility of stocks and the volumes of trading (Antweiler \& Frank, 2004). As such, the shift in the overall perception of Bitcoin can also be attributed to Twitter. Research has shown that there is a high correlation between the Twitter influence users' probability and the probabilities influenced, whilst the majority of the users continue to have a balance in terms of sentiments in both cases (Ranasinghe \& Halgamuge, 2021). Further analysis has shown that on the tweets related to Bitcoin

PICBE | 60 and financial data scrutinized through sentiment analysis, the Twitter sentiment showed a predictive influence for the results of Bitcoin (Sattarov, Jeon, \& Lee, 2020).

\section{Literature Review}

Bitcoin has gained the attention of investors, traders, early adopters, and individuals who want to be a part of a movement, even though social media platforms, such as twitter, a news, and social media platform with over 353 million users, according to a study published in January 2021 on Statista (Statista, 2021).

Therefore, research showed that the number of Bitcoin-related tweets are able to explain the currency's trading volume or returns due to the people's common interest on the topic (Philippas, 2019).

On January 29, 2021, when Elon Musk, a highly influential person and at that time richest person in the world changed his Twitter's account biography to \#bitcoin, the event had a direct impact on the price of Bitcoin, which rose from about USD 32,000 to over USD 38,000 in a few hours and increased its market capitalization by USD 111 billion (Klebnikov, 2021).

A paper focused on how twitter activity influences cryptocurrencies returns proved through event study methodology that the change of Musk's Twitter biography to \#bitcoin resulted in significantly positive cumulative abnormal returns which already amounted to $6.31 \%$ after 30 minutes and culminated at $18.99 \%$ over a period of six hours (Ante, 2021).

Furthermore, research found that an increase in the volume of Bitcoin related tweets yields short-term liquidity increases of the cryptocurrency (Choi, 2020).

Furthermore, an increase in the volume of Bitcoin related tweets yields short-term liquidity increases of the cryptocurrency (Choi, 2020).

A 2020 research analysing social media response in the context of testing the incorporation of a Bitcoin payment platform of five US-based companies proved that there is a positive impact on reputation from the adoption of the new technology.

Using Twitter, data was analyzed to measure how the news on the media platform on the adoption of a new technology may impact the reputation of the companies analyzed, the findings showed a positive correlation on the volume of tech-related tweets and on the sentiment expressed in the tweets themselves, even if patterns of the two effects are different (Caviggioli, 2020).

Research using machine learning through an autoregressive integrated moving average model and a long short-term memory network proved that the AI models using sentiment analysis on tweets containing the keywords "bitcoin" or "btc" predicted the volatility in the values of Bitcoin with a higher degree of accuracy than the model comparing the values without sentiment (Thanekar, 2019).

Much as the research in behavioral economics advocates that emotions have the possibility to alter individual behavior together with the process of decision-making, Twitter, the platform for microblogging, has been increasingly attracting the attention of multiple fields of study, being perceived as a means to analyze economic and social data sets. Interestingly, the platform seems 
to have an influence on its users and on the diffusion of information (Kaminski, 2016). Research on this topic has shown there is a correlation between the activity related to "internet message boards" and the volatility of stocks and the volumes of trading (Antweiler and Frank, 2004).

Our paper aims at finding a niche correlation between tweets and bitcoin traded volume around a specific event detailed in the Objective section. As previous research proved that there is a correlation between big volumes of tweets and bitcoin prices, we look at Tesla's influence following the news of investment in the cryptocurrency as a giant in the tech industry.

PICBE |

\section{Methodology}

On the $8^{\text {th }}$ of February 2021, following Tesla filing its annual $10-\mathrm{K}$ report with the Securities and Exchange Commission, the news that the company invested $\$ 1.5$ billion in the Bitcoin cryptocurrency has taken the tech world, investors and the general public by storm.

The objective of this paper is to analyze the movements of the cryptocurrency trade volume, as well as price before, during and after this event has occurred and if the hype created by social media users on the popular platform, Twitter, following the news spreading, is correlated with volatility of the cryptocurrency.

This research employs three secondary data sets. The first one, regarding the evolution of Bitcoin volume and price wise for the period between 5th of February and 19th of February, has been collected from Yahoo Finance. The second quantitative data set made available on Kaggle (Kaggle, 2021), provides information about the number of tweets with trending \#Bitcoin and \#btc hashtag during the period previously mentioned. The third data set encompasses web searches and news searches having the word grouping Tesla, bitcoin, and Elon Musk from Google Trends (Google Trends, 2021).

When it comes to data analysis, as a processing means, we have used Python where data from the three csv files has been imported, sorted, processed, and displayed in graphs to depict and interpret the findings.

In order to explain the correlation between the social media posts and Bitcoin volatility, simple linear regression has been performed upon the two variables. We considered Bitcoin price to be the dependent variable, and tweets as the independent, explanatory variable.

\section{Results and discussions}

A. Python algorithm and graphical representations of the price and volume of Bitcoin and also the volume of tweets related to the cryptocurrency during the period analyzed

\section{A.1 Importing padas and matplotlib libraries in python}

The first step was to import padas package and gave it the alias pd, which is conventional practice, after that we imported matplotlib through pyplot package and gave it the alias plt in order to visualize date in python.

Code from python

In: import pandas as pd

from matplotlib import pyplot as plt 


\section{A.2 Reading csv file in python}

The second step was to construct a data frame for reading the csv file. The csv file was download from Bitcoin Yahoo Finance profile, and customized for our period analyzed, i.e. starting the $5^{\text {th }}$ of February 2021 and ending with the $19^{\text {th }}$ of February 2021.

Code from python

In: $\quad \mathrm{df}=$ pd.read_csv("BTC_Price.csv")

\section{A.3 Defining date time frame in python}

The third step after reading the csv file in Jupiter notebook was to convert the dates to the framework of datetime in python.

\section{Code from python}

In: $\quad$ df['Date'] = pd.to_datetime(df['Date'],dayfirst=False) df['Date']

$\begin{array}{lll}\text { Out: } & 0 & 2021-02-05 \\ & 1 & 2021-02-06 \\ & 2 & 2021-02-07 \\ 3 & 2021-02-08 \\ & 4 & 2021-02-09 \\ 5 & 2021-02-10 \\ & 6 & 2021-02-11 \\ 7 & 2021-02-12 \\ 8 & 2021-02-13 \\ 9 & 2021-02-14 \\ 10 & 2021-02-15 \\ 11 & 2021-02-16 \\ 12 & 2021-02-17 \\ 13 & 2021-02-18 \\ 14 & 2021-02-19\end{array}$

Name: Date, dtype: datetime64[ns]

A.4 Creating graphical representation regarding the evolution of Bitcoin volume during the $5^{\text {th }}$ and the $19^{\text {th }}$ of February 2021

For displaying the data, we have taken into account the library from python which keeps the date format converted using the code explained above.

Thus, we imported matplotlib. dates, which helped to visualize the chronological dates, by putting the interval between dates equal to one. The figure's size is created based on a scale of 10 to 10 , having a rotation of 45 degrees. The graph contains the columns with the days included in the period analyzed and the volume of Bitcoin transactions. 


\section{Code from python}

In: import matplotlib.dates as mdates

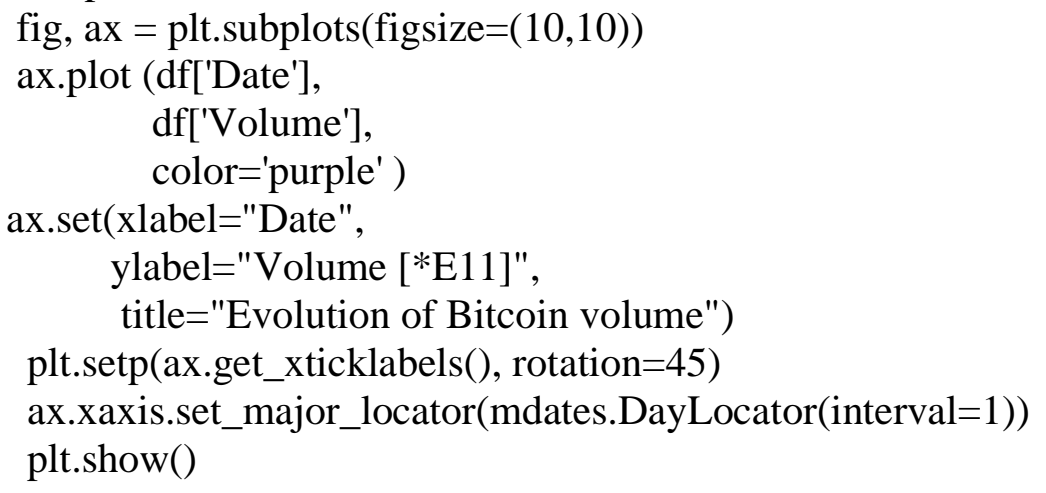

PICBE | 63

\section{Out:}

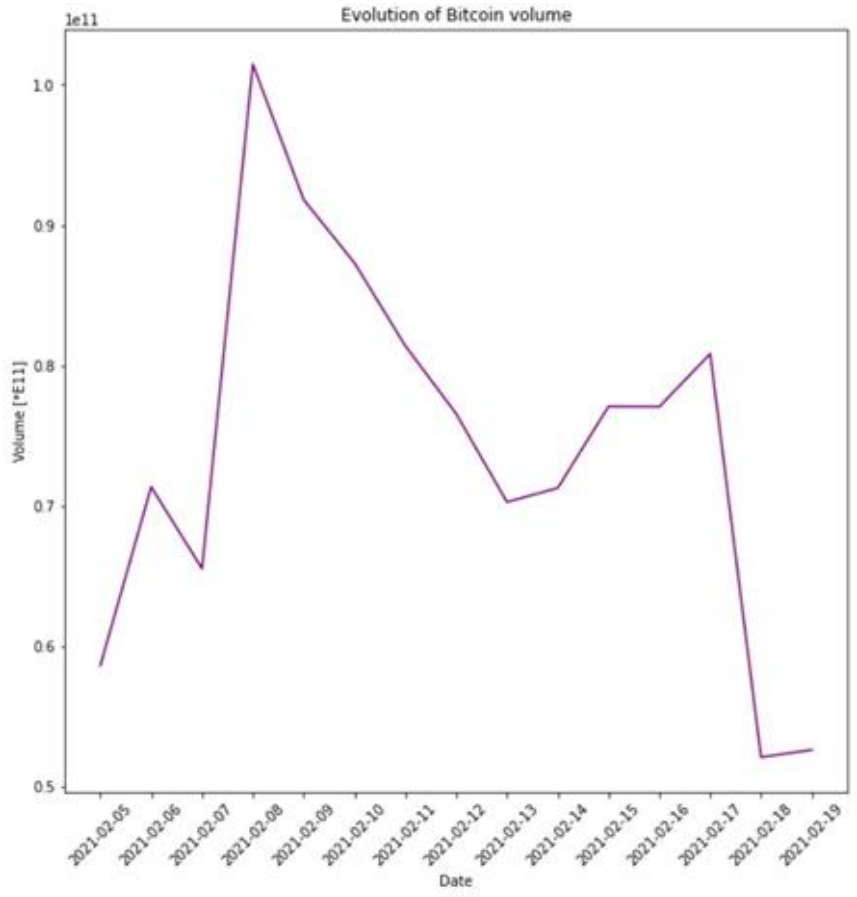

The graph above shows the evolution of Bitcoin volume between the period 05-02-2021 and 19-02-2021. The highly volatile specificity of Bitcoin is depicted in the graph as in the first day of the observations, the volume was quite low and until the mid of the observations period, it rose dramatically, followed by an accelerated decrease in the beginning of the second half of the observation period. The volume then slightly rose, yet in the last days of the observation, it has a steep decrease. 
A.5 Creating graphical representation regarding the evolution of Bitcoin price during the $5^{\text {th }}$ of February 2021 and the $19^{\text {th }}$ of February 2021

The same python code used for determining the evolution of Bitcoin transactions volume was used to visualize the fluctuation of price during the period analyzed.

Code from python

PICBE |

In: $\quad$ fig, ax $=$ plt.subplots $($ figsize $=(10,10))$

ax.plot(df['Date'], df['Adj Close'], color='red' )

ax.set(xlabel="Date", ylabel="Adj Close ", title="Evolution of Bitcoin price")

plt.setp(ax.get_xticklabels(), rotation=45)

ax.xaxis.set_major_locator(mdates.DayLocator(interval=1))

plt.show()

Out:

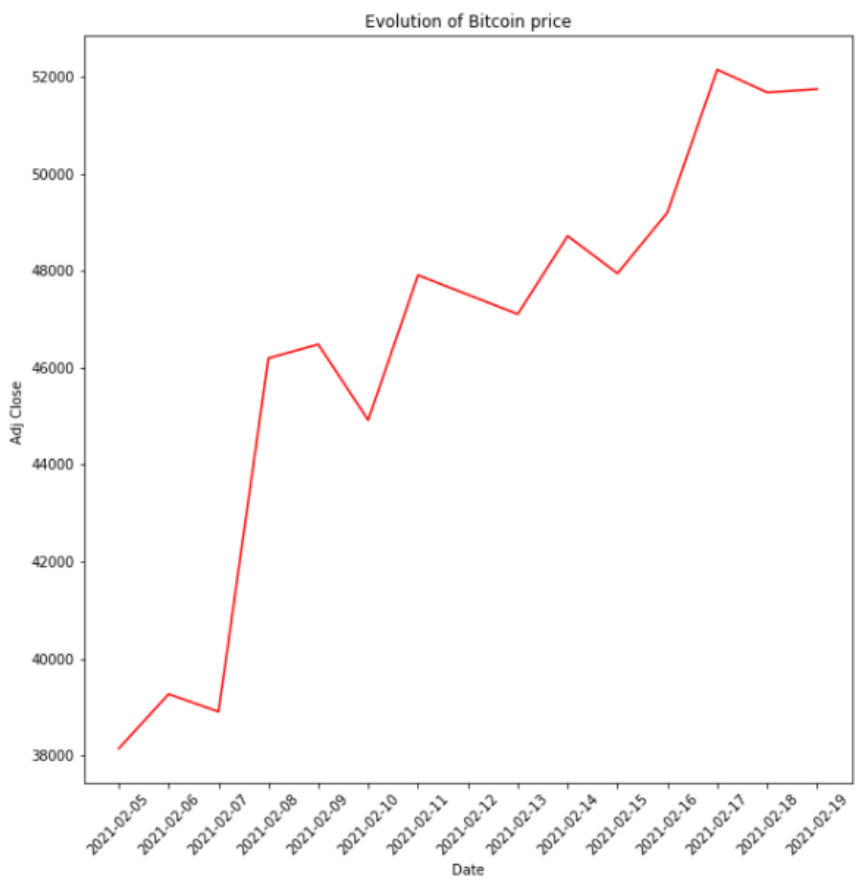

The graph details the evolution of the Bitcoin price. As expected, there are fluctuations in the crypto-currency's price, even over a period of fifteen days. The price substantially rose in the first part of the analyzed period and it maintained the ascending trend until the end of the period, despite the frail contractions that occurred. Overall, it appears the price of Bitcoin increased by nearly $37 \%$, from USD 38 000, up to approximately USD 52000 at the end of the period. 
The steepest increase can be identified between 8th and 9th of February, culminating with a $22.7 \%$ increase at the end of the trading day on the 9th, with the price of USD 47.899.

\section{A.6. Counting the number of tweets based on the days when they were posted on Twitter}

Due to the fact that in the csv file downloaded from Kaggle website, there was not a column which contains the volume of tweets having the hashtag Bitcoin or BTC, but the csv contains when the tweets were posted. Thus, we counted in python the volume of tweets using the code mentioned below (Kaggle, 2021).

\section{Code from python}

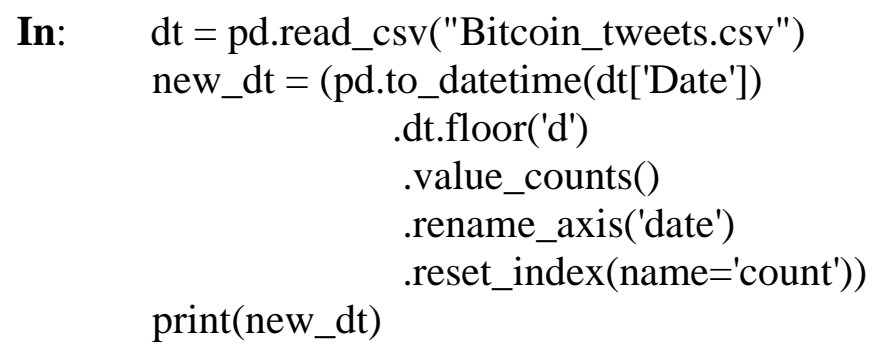

Out: $\quad \begin{array}{lcc}\text { date } & \text { count } \\ 0 & 2021-02-08 & 5647 \\ 1 & 2021-02-15 & 4651 \\ 2 & 2021-02-19 & 4521 \\ 3 & 2021-02-09 & 4350 \\ 4 & 2021-02-14 & 4009 \\ 5 & 2021-02-10 & 3526 \\ 6 & 2021-02-06 & 3278 \\ 7 & 2021-02-07 & 3030 \\ 8 & 2021-02-13 & 2285 \\ 9 & 2021-02-18 & 2254 \\ 10 & 021-02-05 & 1694\end{array}$

A.7 Creating graphical representation as per the volume of tweets regarding Bitcoin during the $5^{\text {th }}$ and the $19^{\text {th }}$ of February 2021

After determining the volume of tweets posted during the period analyzed, we created the graphical representation based on the same code written for the above two graphics.

\section{Code from python}

In: fig, $\mathrm{ax}=$ plt.subplots $($ figsize $=(10,10))$

$$
\begin{array}{r}
\text { ax.bar(new_dt['date'], } \\
\text { new_dt['count'], } \\
\text { color='blue' ) } \\
\text { ax.set(xlabel="Date", }
\end{array}
$$




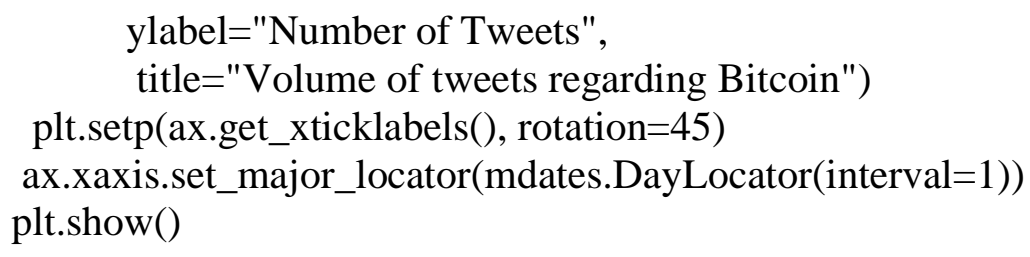

Out:

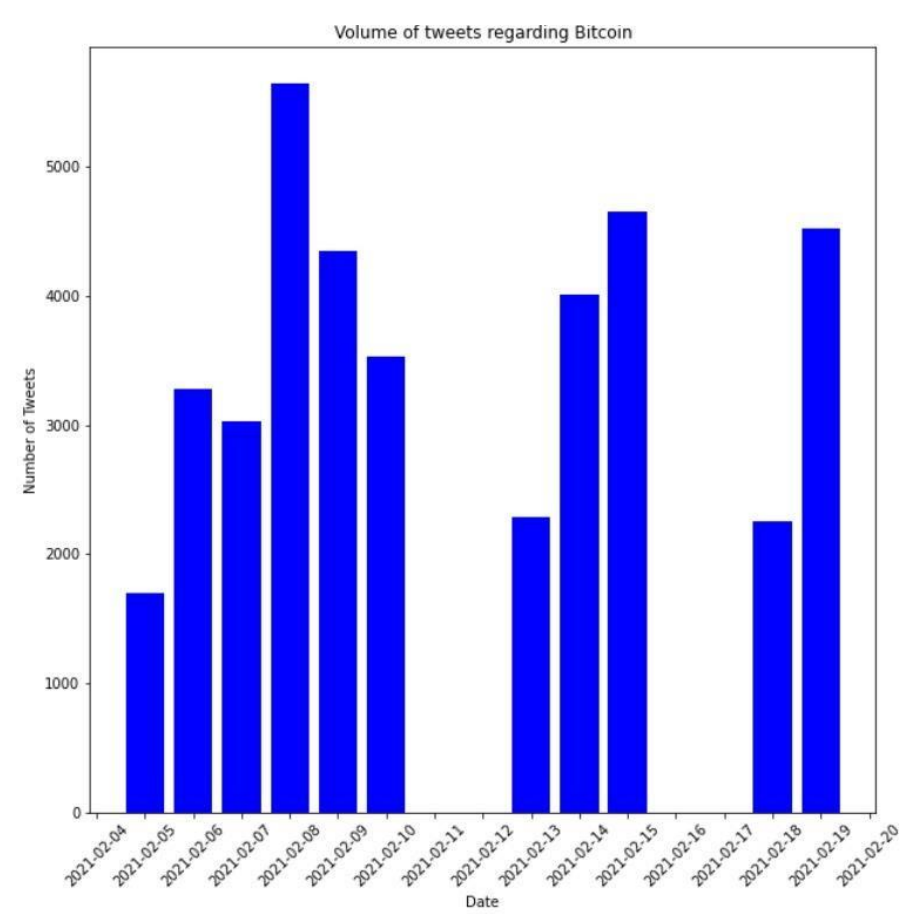

The peak of tweets regarding bitcoin was identified as seen in the graph above on the $8^{\text {th }}$ of February, when Tesla announced its investment and when the price of bitcoin saw the sharpest increase. (Google Trends, 2021)

B. Python algorithm and graphical representations of web searches and news searches having the word grouping tesla, bitcoin and Elon Musk from Google Trends

\section{B.1 Importing csv file and reading the data as table in python for web searches}

Before creating the graphs, we read the data in python from csv through a table which contains the numbers of rows, columns and the head of the table in order to create a correlation between the csv data format and the data needed to be visualized in python.

\section{Code from python}

In: df $1=$ pd.read_csv("data_1.csv", index_col=0, parse_dates=True $)$ df.head(15) 
Out:

\begin{tabular}{rrrr} 
& Day & Tesla-bitcoin (web search) & Elon Musk (web search) \\
\hline 0 & $2021-02-05$ & 2 & 50 \\
1 & $2021-02-06$ & 1 & 60 \\
2 & $2021-02-07$ & 1 & 61 \\
3 & $2021-02-08$ & 21 & 78 \\
4 & $2021-02-09$ & 13 & 59 \\
5 & $2021-02-10$ & 6 & 53 \\
6 & $2021-02-11$ & 6 & 50 \\
7 & $2021-02-12$ & 5 & 50 \\
8 & $2021-02-13$ & 3 & 45 \\
9 & $2021-02-14$ & 2 & 42 \\
10 & $2021-02-15$ & 3 & 43 \\
11 & $2021-02-16$ & 4 & 41 \\
12 & $2021-02-17$ & 5 & 39 \\
13 & $2021-02-18$ & 4 & 42 \\
14 & $2021-02-19$ & 4 & 43
\end{tabular}

B.2 Creating graph related to the web searches on Google having the word groupings tesla, bitcoin and Elon Musk

The syntax df1.plot() print the data defined below in the code used to generate the table in python.Code from python

In: df1.plot( $)$

Out: <AxesSubplot:xlabel='Day'>

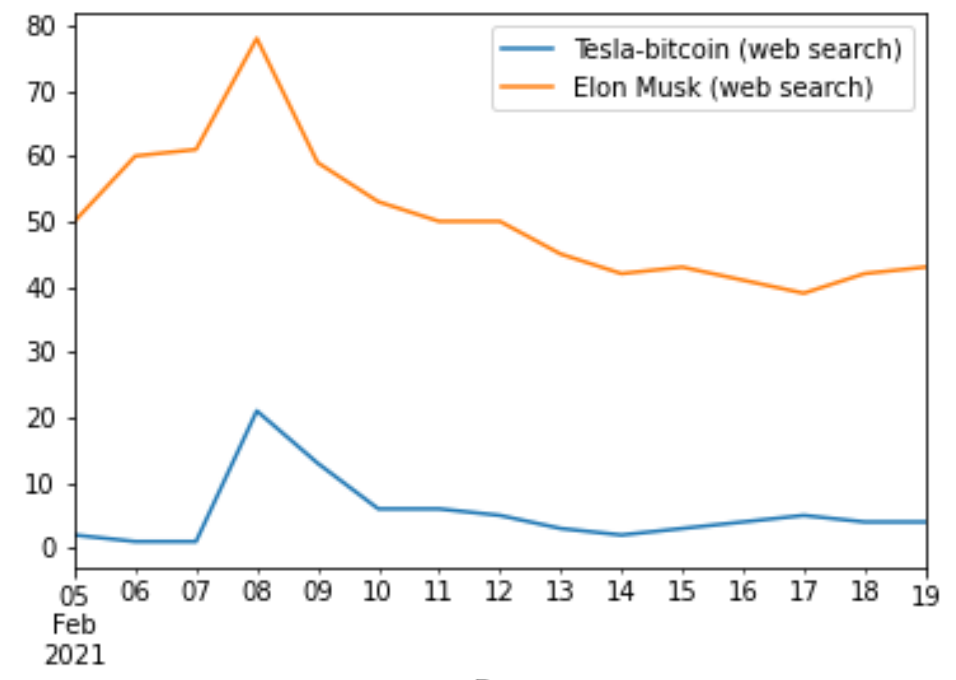

Day

In the graph above, it can be outlined the peak of the web searches having the word groupings tesla, bitcoin, and Elson Musk on $8^{\text {th }}$ of February 2021. The same trend of the tweets containing posts related to bitcoin, Elon Musk, was followed on Twitter. The web searches with 
Elon Musk are higher than those with bitcoin-tesla due to the announcement made by the Tesla CEO.

B.3 Importing csv file and reading the data as table in python for news searches

For this step, we focused on the same python code for generating tables and graphs related to news searches containing bitcoin, tesla and Elon Musk words.

Code from python

In: df2 = pd.read_csv("data_2.csv", index_col=0, parse_dates=True) df.head(15)

Out:

\begin{tabular}{rrr}
\multicolumn{2}{r}{ Tesla-bitcoin (news search) } & Elon Musk (news search) \\
Day & 0 & 28 \\
\hline $2021-02-05$ & 0 & 62 \\
$2021-02-06$ & 0 & 34 \\
$2021-02-07$ & 85 & 81 \\
$2021-02-08$ & 0 & 35 \\
$2021-02-09$ & 13 & 48 \\
$2021-02-10$ & 0 & 50 \\
$2021-02-11$ & 0 & 32 \\
$2021-02-12$ & 0 & 31 \\
$2021-02-13$ & 0 & 36 \\
$2021-02-14$ & 0 & 41 \\
$2021-02-15$ & 4 & 17 \\
$2021-02-16$ & 4 & 44 \\
$2021-02-17$ & 0 & 24 \\
$2021-02-18$ & 0 & 30 \\
$2021-02-19$ & &
\end{tabular}

B.4 Creating graph related to the news searches on Google having the word groupings tesla, bitcoin and Elon Musk

Code from python

In: $d f 2 \cdot p \operatorname{lot}()$ 
Out:

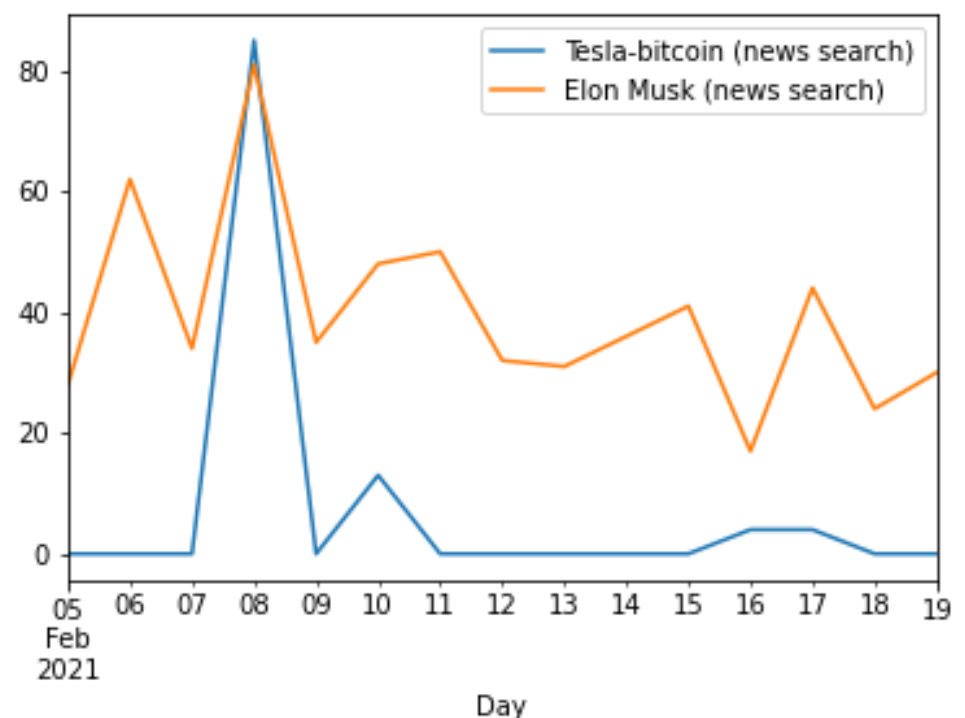

PICBE | 69

Figure 7. Graphical representation of news searches

Source: Authors' own research.

The peak of news searches with the same word grouping as the graph presented above, was also on $8^{\text {th }}$ of February 2021. The trend of news searches has the same impact as per the interest of the people searching on Google when Tesla announced its investment.

\section{Python algorithm and graphical representations of linear regression \\ C.1 Importing packages and classes in python}

In order to start implementing and plotting linear regression, we imported matplotlib package for data visualization and padas for reading the data in csv format. The class sklearn.linear_model was imported for performing the linear regression and for making predictions accordingly.

\section{Code from python}

In: $\quad$ import matplotlib.pyplot as plt \# To visualize

import pandas as pd \# To read data

from sklearn.linear_model import LinearRegression

\section{C.2 Reading csv in python and determining the values of dependent and independent variables}

The second step was to define the data from csv and to determine the regressor $\mathrm{x}$ and the predictor $\mathrm{y}$ for the linear regression. Thus, in our case, the regressor is the independent variable $\mathrm{x}$, which refers to the values of the volume related to tweets with the hashtag Bitcoin or BTC, while the dependable variable, $y$, indicates the adjusted value of the Bitcoin price during our period analyzed. We used the syntax values.reshape $(-1,1)$ for converting values into a numpy array, while -1 represents the calculation of the rows dimension with one column. 


\section{$\underline{\text { Code from python }}$}

In: data $=$ pd.read_csv('data.csv')

$\mathrm{X}=$ data.iloc[:, 0].values.reshape $(-1,1)$

$\mathrm{Y}=$ data.iloc[:, 1]

$\operatorname{print}(\mathrm{X})$

$\operatorname{print}(\mathrm{Y})$

PICBE |

Out: [[3278]

[3030]

[5647]

[4350]

[3526]

[ 0$]$

[ 0$]$

[2285]

[4009]

[4651]

[ 0$]$

[ 0$]$

[2254]

[4521]]

$0 \quad 39266$

138903

246196

$3 \quad 46481$

444918

547909

$6 \quad 47505$

$7 \quad 47106$

$8 \quad 48717$

947945

$10 \quad 49200$

1152149

1251680

$13 \quad 51747$

Name: 38144, dtype: int64

C.3 Creating a model for linear regression and adjusted it with the data for our analysis

The syntax presented below creates the object for the class.

Code from python

In: model $=$ LinearRegression ()$\cdot$ fit $(X, Y)$ 
C.4 Determining coefficient of determination, intercept and slope for the linear regression model

Code from pyhton

In: $r \_s q=$ model.score $(X, Y)$

print('coefficient of determination:', r_sq)

PICBE |

Out: coefficient of determination: 0.05868858652022213

The coefficient of determination $\mathrm{R}^{2}$ is determined by applying the code syntax .score() based on the regressor and predictor values.

In: print('intercept:', model.intercept_)

Out : intercept: 48446.47020264655

The .intercept, represents the $\beta 0$, while the .coef_represents the $\beta 1$ and both are the attributes of the model.

In: print('slope:', model.coef_)

Out: slope: [-0.49342449]

The code explained above, illustrates how to determine $\beta_{0}$ and $\beta_{1}$. The intercept_is a scalar, while the .coef_is an array.

The value of the intercept, $\beta_{0}=48446.47$ shows the point where the estimated regression line crosses the y axis. $\beta_{1}$ equals with -0.49 determines the slope of the estimated regression line.

\section{C.5 Making predictions based on the linear regression model}

Code from python

The syntax .predict(), shows the regressor as the argument and gets the corresponding predicted response.

In: $Y \_p r e d=$ model.predict $(X)$ print('predicted response:', Y_pred, sep=''n')

Out : predicted response: [46829.02474075 46951.3940130345660.1021357346300.0736928 46706.6554684648446 .4702026548446 .4702026547318 .99525437 46468.3314421946151 .5529228148446 .4702026548446 .47020265 $47334.2914134146215 .69810586]$ 


\section{C.6 Graphical representation of the linear regression}

\section{$\underline{\text { Code from python }}$}

The syntax of plotting used presents the visualization results computed in the below lines of the pyhton code, which shows the relationship between the dependent variable (Bitcoin price) and the independent variable (Tweets with the hashtag Bitcoin or BTC).

PICBE |
In: $\quad$ plt.scatter(X, Y)
plt.plot(X, Y_pred, color='green')
plt.show()

Out:

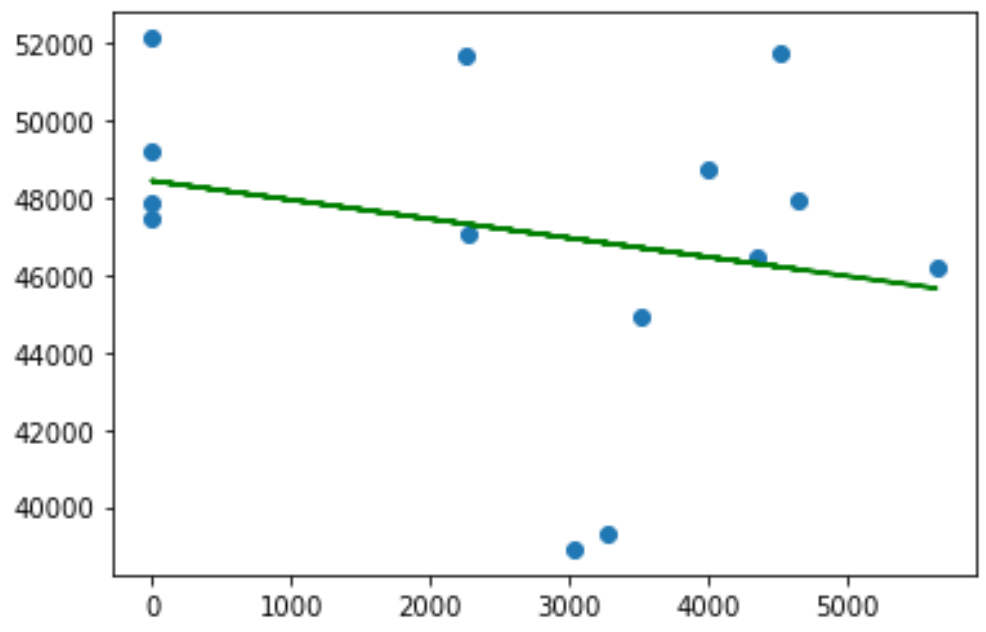

The $\mathrm{R}^{2}$ and standard error of regression are measures of how close the values of $\mathrm{Y}$ (Bitcoin price) are to the estimated regression line. The regression, $R^{2}$ is the fraction of the sample variance of $\mathrm{Y}$, predicted by $\mathrm{X}$ and it is an indicator of the goodness of fit (Linear Regression - Detailed View, 2021).

$\mathrm{R}$ Square is 0,058 , meaning $5,8 \%$ out of Bitcoin price variation is due to variation in tweets, while holding the other factors constant. There is a low intensity relation between Bitcoin price and tweets

The trend line coupled with the slope sign provides insights on the relationship between the two variables. The slope $\beta_{1}$ represents the change in $Y$ associated with a unit change in $X$. Thus, it shows a negative correlation between Bitcoin price and tweets, if one variable increases, the other follows the opposite trend.

Residuals are spread, errors are appearing above and below the line of best fit. 


\section{Conclusion}

This research has been centered around a big event - Tesla's tremendous investment in Bitcoin and the possibility to accept it as payment in the near future. The results gathered in the aftermath of the data sets analysis have revealed two main points.

The first one, is that the event had a huge impact on people's interest on Bitcoin and the topic. There is no doubt that social media movements following the news have bloomed that day the graphs of web searches and news searches having the word grouping tesla, bitcoin and Elon

PICBE | Musk, as well as the number of tweets using btc and bitcoin hashtag peak on the same day the event occurs. The fear of missing out is a common symptom that has not spared the cryptocurrencies domain. Once considered the domain of odd one outs, right now a hot and hyped topic, Bitcoin tends to become a mainstream subject, as people start to exhibit interest in engaging more in research, discussions, and debates regarding the cryptocurrency. But is it enough? Is the social media craze and talk really a booster to Bitcoin's volatility? Do people just talk to be a part of something bigger and not miss the wave or are they really investing?

These questions bring us to the second point, with reference to the linear regression results. The results previously displayed show little to no correlation between Twitter posts and the Bitcoin price during the period when the big news took place. Moreover, the relationship between the two is negative.

The specific objective of this research has narrowed the period for which data had to be gathered, therefore the short data frame might represent a jeopardizing factor to the research. For further, more extensive groundwork a larger set of alike events and a lengthier period should be considered.

Another pitfall can be the decision to use secondary data sets only. The degree of control upon the quality of the data gathered is higher in the case of primary data, compared to secondary one. Using secondary data can raise validity and reliability issues. Moreover, secondary data may not perfectly fit the needs of the researcher since the scope for which was collected in the first place is different.

\section{References}

Ante, L. (2021). How Elon Musk's Twitter Activity Moves Cryptocurrency Markets (16). SSRN Electronic Journal.

Antweiler, W., \& Frank, M. (2004). Is all that talk just noise? The information content of Internet stock message. Journal of Finance 59,3.

Caviggioli, F. L. (2020). Technology adoption news and corporate reputation: sentiment analysis about the introduction of Bitcoin, Journal of Product \& Brand Management 29(7), pp. 877-897.

Chena, C., Liub, L., \& Zhaoc, N. (2020). Fear Sentiment, Uncertainty, and Bitcoin Price Dynamics: The Case of COVID-19. Emerging Markets Finance and Trade.

Choi (2020). Investor attention and bitcoin liquidity: Evidence from bitcoin tweets. Finance Research Letters.

Davis, A. B., \& Petasis, A. (2021). To Bitcoin or Not to Bitcoin; Exploring Consumer Perceptions. The International Journal of Business Management and Technology, 5(1).

Forbes (2021). What Publicly Traded Companies Have Bitcoin On Their Balance Sheet - And Why. Retrieved from https://www.forbes.com/sites/rogerhuang/2021/01/12/what-publiclytraded-companies-have-bitcoin-on-their-balance-sheet---and-why/?sh=4a1df4f54564. 
Google Trends (2021, February). Retrieved from https://trends.google.com/trends/explore?date= today\%201-m\&q=bitcoin\%20tesla,\%2Fm\%2F03nzf1.

Kaggle (2021, February 2). Retrieved from https://www.kaggle.com/kaushiksuresh147/bitcointweets.

Kaminski, J. (2016). Nowcasting the Bitcoin Market with Twitter. MIT Media Lab.

Kaushal, P. K., Bagga, A., \& Sobti, R. (2017). Evolution of Bitcoin and Security Risk in Bitcoin. International Conference on Computer, Communications and Electronics (Comptelix) Manipal University Jaipur, Malaviya National Institute of Technology Jaipur \& Irisworld.

PICBE | 74

Klebnikov, S. (2021). Elon Musk Is The Richest Person In The World - Again. Retrieved from https://www.forbes.com/sites/sergeiklebnikov/2021/01/14/elon-musk-is-the-richestperson-in-the-world-again/.

Leath, J. (2019). Is Bitcoin Reminiscent of Past Bubbles? University of Tennessee at Chattanooga, Student Research, Creative Works. [online] retrieved from https://scholar.utc.edu/honorstheses/200/ [Accessed 24 February 2021].

Linear Regression - Detailed View (2021). Retrieved from Towards Data Science: https://towardsdatascience.com/linear-regression-detailed-view-ea73175f6e86.

Okhuese, A. V. (2017). Introducing Cryptocurrency. Reads Capital, Schemas Group.

Philippas, D. R. (2019). Media attention and Bitcoin prices. 30, 37-43. Retrieved from https:// doi.org/10.1016/j.frl.2019.03.031.

Ranasinghe, H., \& Halgamuge, M. (2021). Twitter Sentiment Data Analysis of User Behavior on Cryptocurrencies: Bitcoin and Ethereum. In Analyzing Global Social Media Consumption.

Reuters (2021). MicroStrategy to borrow $\$ 600$ million to buy more bitcoin. Retrieved from https://www.reuters.com/article/us-crypto-currency-microstrategy/microstrategy-toborrow-600-million-to-fund-bitcoin-buying-idINKBN2AG1LA.

Sattarov, O., Jeon, H., \& Lee, J. (2020). Forecasting Bitcoin Price Fluctuation by Twitter Sentiment Analysis. 2020 International Conference on Information Science and Communications Technologies (ICISCT).

Segendorf, B. (2014). What is Bitcoin?.

Shen, D. U. (2019). Does twitter predict Bitcoin?. 174, 118-122. Retrieved from https://doi.org/ 10.1016/j.econlet.2018.11.007.

Thanekar, A. S. (2019). Bitcoin Movement Prediction Using Sentimental Analysis of Twitter Feeds. (I. J. Engineering, Ed.) 7(2), pp.148-152.

Work, C. 2.-B. (2019). How “True Bitcoiners" Work on Reddit to Maintain Bitcoin. CHI 2019 Late-Breaking Work.

YahooFinance (2021). Bitcoin USD (BTC-USD). Retrieved from https://finance.yahoo.com/ quote/BTC-USD?p=BTC-USD\&.tsrc=fin-srch.

Statista (2021). Most used social media 2020 | Statista. [online] Retrieved from: https://www. statista.com/statistics/272014/global-social-networks-ranked-by-number-of-users/ [Accessed 22 February 2021]. 